\title{
ELEMENTOS Y CARACTERÍSTICAS DEL MATERIAL IMPRESO QUE FAVORECEN LA FORMACIÓN Y EL APRENDIZAJE A DISTANCIA EN LA UNED (Sistematización de características y resultados globales)
}

\section{ELEMENTS AND CHARACTERISTICS OF THE PRINTED MATTER THAT PROMOTE EDUCATION} AND DISTANCE LEARNING IN THE UNED (Systematization of characteristics and global results)

\author{
Giuseppa D’Agostino \\ Johanna Meza \\ Alejandra Cruz \\ Universidad Estatal a Distancia (Costa Rica)
}

\section{RESUMEN}

El artículo que aquí se presenta tiene su origen en un proyecto investigativo múltiple orientado hacia la construcción del conjunto de elementos y características deseables en los textos didácticos de la UNED de Costa Rica y hacia la determinación de aquellos que de esos aspectos le resultan al estudiante, de dicha Institución, de mayor utilidad para su aprendizaje y formación académica. Además, se aporta una síntesis de las dos primeras partes del trabajo, las cuales corresponden a las bases teóricas, la construcción de los perfiles de las áreas temáticas y los resultados macro del proyecto.

\begin{abstract}
This article is the result of a multiple research project orientated to the construction of desirable elements and characteristics in the didactic books produced at UNED (Costa Rica) and the detection of which are the most useful ones for the learning process and academic formation of the student. The paper also presents a summary of the two first parts of the work: the theoretical and the construction of the thematic profile areas, and the macroresults of the project.
\end{abstract}


Giuseppa D `Agostino, Johanna MeZa y Alejandra Cruz

ELEMENTOS Y CARACTERÍSTICAS DEL MATERIAL IMPRESO QUE FAVORECEN LA FORMACIÓN Y EL APRENDIZAJE A DISTANCIA EN LA UNED. SiSTEMATIZACIÓN DE CARACTERÍSTICAS Y RESULTADOS GLOBALES

\section{PRESENTACIÓN}

El presente trabajo tiene como base de referencia un proyecto global de investigación iniciado por las autoras, funcionarias de la Universidad Estatal a Distancia de Costa Rica, a finales del año 2000 y completado en el curso del año 2003 (Meza y otras, 2003).

El propósito medular de dicho proyecto consiste en identificar el conjunto de elementos y características que debe poseer el texto didáctico para favorecer el estudio en el sistema educativo de la UNED y determinar cuáles de esos elementos y características tienen una mayor influencia en el aprendizaje y en el desarrollo académico del estudiante de dicha institución educativa.

El proyecto completo está compuesto por cuatro estudios investigativos, a saber: El primero abarca una revisión de las bases teóricas de la educación a distancia y sus implicaciones en la elaboración de los materiales didácticos impresos. También, brinda los lineamientos y el desarrollo histórico de la producción de materiales impresos en la UNED. Para ello, se recopilan y sistematizan los aportes que sobre dicha producción han planteado en diversos documentos, los funcionarios de la institución, expertos en la producción de materiales didácticos impresos.

El segundo se relaciona directamente con la sistematización y construcción de elementos (áreas temáticas), características y atributos que en opinión de los profesionales de la UNED consultados, aseguran un nivel óptimo de calidad del texto impreso para el sistema de estudio a distancia de la misma Institución.

El tercer estudio se dirige hacia la corroboración y diferenciación de esos elementos y características de importancia, con graduados de las distintas escuelas de la UNED.

El cuarto y último trabajo consiste en elaborar con base en los resultados que se desprenden de los estudios anteriores, un cuestionario que se aplica a una muestra de 1900 estudiantes, y en discutir, analizar e interpretar los resultados obtenidos.

Estos estudios son relativamente independientes entre sí, pero se entrelazan en un cuerpo teórico metodológico y de aplicación, con el fin de lograr realmente el propósito antes señalado. En seguida, se presenta, por razones de espacio, solamente una somera síntesis de la primera y segunda parte del proyecto global, que constituyen la base para la puesta en práctica de la cuarta parte. De esta última, se 
Giuseppa D `Agostino, Johanna MeZa y Alejandra Cruz

ELEMENTOS Y CARACTERÍSTICAS DEL MATERIAL IMPRESO QUE FAVORECEN LA FORMACIÓN Y EL APRENDIZAJE A DISTANCIA EN LA UNED. SiSTEMATIZACIÓN DE CARACTERÍSTICAS Y RESULTADOS GLOBALES

aportan, nada más que, una breve descripción de la metodología y de los resultados más destacados.

Todo lo anterior, no sin antes señalar que el estudio, en su conjunto, es de tipo exploratorio y sus metas prioritarias son las de descubrir elementos del medio escrito significativos para el estudiante de la UNED, efectuar algunas correlaciones entre diversas situaciones y generar conocimiento al respecto. Conforme con la naturaleza del estudio, la metodología prevaleciente es de tipo cualitativo con énfasis en la interpretación, comparación y contraste entre las construcciones de las investigadoras y las de los distintos actores que intervienen en el estudio. De aquí la utilización de estrategias como el análisis de documentos oficiales, la triangulación de la información obtenida desde diversas fuentes y las entrevistas individuales y grupales. También, se recurre a técnicas de tipo cuantitativo, como es el caso de la encuesta a una muestra de estudiantes donde se aplica un cuestionario y se analizan y correlacionan los datos obtenidos.

\section{BASES TEÓRICAS DE LA EDUCACIÓN A DISTANCIA, SUS IMPLICACIONES EN LA ELABORACIÓN DE MATERIALES DIDÁCTICOS Y PAUTAS DE PRODUCCIÓN DE MATERIALES IMPRESOS EN LA UNED.}

En esta primera fase del proyecto se atienden los aspectos teóricos y el desarrollo histórico de la producción de materiales impresos en la UNED. Ello con la finalidad de inferir y enmarcar los lineamientos teóricos referenciales y también para sistematizar la experiencia de la UNED, a manera de memoria histórica. Seguidamente se presenta una síntesis de ambas partes.

\section{Implicación de las perspectivas teóricas en la elaboración de los materiales didácticos.}

Se realiza una revisión teórica sobre la educación a distancia y sus implicaciones en la elaboración de materiales didácticos impresos, que sirve de fundamento para contextualizar y sistematizar el trabajo realizado.

Desde una perspectiva global, las concepciones teóricas (teorías de la industrialización, de la independencia y autonomía, de la interacción y comunicación, de la comunicación bidireccional y de la equivalencia), a pesar de sus divergencias, apuntan hacia las características de dos elementos esenciales de la modalidad 
Giuseppa D `Agostino, Johanna MeZa y Alejandra Cruz

ELEMENTOS Y CARACTERÍSTICAS DEL MATERIAL IMPRESO QUE FAVORECEN LA FORMACIÓN Y EL APRENDIZAJE A DISTANCIA EN LA UNED. SiSTEMATIZACIÓN DE CARACTERÍSTICAS Y RESULTADOS GLOBALES

a distancia, a saber: el estudiante destinatario de la formación y la enseñanza mediatizada (D’Agostino, 2000: 219).

- El estudiante destinatario de la formación a distancia viene identificado, en términos amplios, dentro de las teorías aquí referidas, como alumno a distancia con características de aprendizajes propias del adulto. Como tal (adulto) se parte del supuesto que aprende más fácilmente mediante la experiencia; ello es en ambientes educativos que le permitan practicar y aplicar en su entorno lo que está aprendiendo. También, se considera que, en calidad de adulto, tiene mayor interés en aprender y desarrollar competencias significativas y funcionales para su situación contextual.

- La enseñanza mediatizada: Es el segundo elemento común a los planteamientos teóricos antes esbozados y constituye el meollo y la peculiaridad de la educación a distancia, que se efectúa, esencialmente, a través "de distintos medios y recursos didácticos que favorecen la comunicación y el estudio individualizado mediante la interrelación y el diálogo. En el estudio a distancia si bien, de ser posible se da la comunicación cara a cara, esta, en gran medida es prevalentemente escrita, mecánica y electrónica" (D’Agostino 2000, p. 219). Esto significa que el hecho pedagógico se lleva a cabo esencialmente, mediante el uso de materiales aptos para el autoaprendizaje en un contexto no convencional.

Las anteriores connotaciones del alumno destinatario y de la enseñanza mediatizada, que se desprenden de las perspectivas teóricas dela educación a distancia y de la concepción cognoscitiva del aprendizaje (significatividad, independencia, continuidad, utilidad), constituyen las bases desde las cuales se obtienen una serie de señalamientos y prescripciones que orientan el diseño y la elaboración de los materiales educativos para el estudio a distancia. Estos conjuntos de señalamiento se direccionan hacia dos líneas congruentes de interés, a saber:

- Los materiales deben ser representativos del estado actual de la disciplina sobre la que vierten y ser aptos para el autoaprendizaje. Motivar al estudiante y exponer los contenidos mediante un discurso didáctico, en el nivel de dificultad adecuado para el programa y la población meta. También, deben emplearse estrategias pedagógicas interactivas, favorecedoras de experiencias educacionales que 
Giuseppa D `Agostino, Johanna Meza y Alejandra Cruz

ELEMENTOS Y CARACTERÍSTICAS DEL MATERIAL IMPRESO QUE FAVORECEN LA FORMACIÓN Y EL APRENDIZAJE A DISTANCIA EN LA UNED. SiSTEMATIZACIÓN DE CARACTERÍSTICAS Y RESULTADOS GLOBALES

impulsen al estudiante adulto a comprometerse de manera activa en su formación. (D'Agostino 2000).

- La segunda línea de señalamientos y reflexiones hace referencia a la necesidad de utilizar diversos medios y por ende varios materiales pedagógicos, pero no de manera separada tal como se dio en las primeras etapas de la Educación a Distancia (Holmberg 1995, Casas 1999) sino integrados como un todo unitario, en función de las metas educacionales en común. El diseño integrado de este conjunto de materiales debe favorecer la interacción educativa, la comunicación bidireccional y ambientes de aprendizajes creativos y participativos. Además, este diseño debe procurar la flexibilidad para permitir la actualización permanente de la propuesta educacional y alternativas de itinerarios de formación ( $D^{\prime}$ Agostino 2000). En este sentido, destacan las ventajas del uso de las nuevas tecnologías de la comunicación, que las Instituciones a Distancia incorporan en sus programas educativos, de manera paulatina, según lo permite sus propias realidades y las de su población meta.

Desde las anteriores perspectivas, y en vista de los supuestos teóricos que las fundamentan, un material didáctico para la formación a distancia y, en nuestro caso, un material impreso, debe cumplir con ciertas condiciones (Rowntree 1990, 1998; Misanchuk 1992; García Aretio 2001; Mac Donald-Ross 1995; Hidalgo y otros 1998; Barrantes R., 2000; Holmberg B. 1985, 1995; Garrison, 1995), que garanticen su calidad formativa y su efectividad para el autoaprendizaje. Dentro de estas condiciones destacan los siguientes:

- Estructurar el material y organizar el desarrollo interno de la temática de manera clara, sistemática e integradora.

- Proponer objetivos educacionales que contemplen las distintas competencias por desarrollar con el estudio de la propuesta pedagógica planteada mediante el material y que despierten el interés del estudiante.

- Desarrollar contenidos que den cuenta del estado actual del área disciplinaria y profesional de la que tratan y que sean rigurosos y relevantes para la propuesta educativa y la población meta a las que se destinan. Su grado de profundidad y de extensión debe ser, también, congruente con las exigencias y el nivel de esa misma propuesta de formación. 
Giuseppa D `Agostino, Johanna MeZa y Alejandra CRuZ

ELEMENTOS Y CARACTERÍSTICAS DEL MATERIAL IMPRESO QUE FAVORECEN LA FORMACIÓN Y EL APRENDIZAJE A DISTANCIA EN LA UNED. SiSTEMATIZACIÓN DE CARACTERÍSTICAS Y RESULTADOS GLOBALES

- Enlazar la información nueva con el conocimiento y la experiencia vivencial del alumno destinatario y con los puntos temáticos desarrollados con antelación.

- Utilizarestrategiasdeenseñanzaydeaprendizajeyrecursosacadémicos que faciliten, motiven y estimulen al estudiante a autoaprender y a interactuar con la información, procesarla y transformarla en un conocimiento personal.

- Privilegiar actividades y ejercicios que incentiven en el estudiante la crítica, la reflexión y la transferencia y aplicación de lo aprendido a situaciones y problemas propios de su entorno contextual y profesional.

- Usar un lenguaje claro, específico y coloquial.

- Sugerir formas para complementar, ampliar y profundizar lo estudiado.

- Incorporar actividades, preguntas, guías y parámetros para la autoevaluación y la autorregulación del aprendizaje.

- Usar los recursos técnico-gráficos de manera apropiada desde las perspectivas técnica y académica.

- Favorecerundiseñoversátildelmaterial,evitandolasobrestructuración, incluyendo distintos tipos de actividades y generando diversos caminos para lograr los propósitos de formación.

\section{Sistematización de aportes sobre la producción de materiales impresos planteados en documentos de funcionarios de la institución, expertos en la producción de materiales didácticos impresos.}

La segunda parte de la revisión de las bases teóricas se refiere a la sistematización de un conjunto de documentos que, sobre la producción de materiales didácticos impresos, han elaborado diferentes profesionales que tienen relación con esta producción, desde la misma apertura de la institución en 1977 hasta la fecha. Estos documentos tratan de determinar las pautas necesarias para que los estudiantes de la UNED reciban un producto de calidad acorde con sus necesidades y con los requerimientos de la sociedad, de la institución, del programa académico y del aprendizaje independiente.

El análisis de estos documentos permitió caracterizar tres etapas en la evolución por la que ha pasado la UNED en la producción de sus materiales didácticos impresos. 
Giuseppa D `Agostino, Johanna Meza y Alejandra Cruz

ELEMENTOS Y CARACTERÍSTICAS DEL MATERIAL IMPRESO QUE FAVORECEN LA FORMACIÓN Y EL APRENDIZAJE A DISTANCIA EN LA UNED. SiSTEMATIZACIÓN DE CARACTERÍSTICAS Y RESULTADOS GLOBALES

La primera se inicia en 1979 con la apertura de la universidad y la producción de sus primeros materiales didácticos impresos. Sus lineamientos siguen, prevalentemente, la corriente conductista. Por lo tanto, la producción se basa en aspectos como la importancia de los objetivos conductuales, el refuerzo continuo, los ejercicios de autoevaluación, los cuales deben evaluar el logro de cada uno de esos objetivos, y el contenido se desarrolla de acuerdo con los objetivos preestablecidos, de manera bastante lineal y prescriptiva.

La segunda etapa se puede denominar de transición y se inicia después del Primer Congreso Universitario (1988); se nota un incremento en los aportes de los especialistas de la UNED para tratar de ofrecer unidades didácticas apropiadas para la educación a distancia y se inicia el cuestionamiento del enfoque conductista, al tomar mayor fuerza la corriente cognitiva-constructivista del aprendizaje. Comienzan a desarrollarse ideas tales como la importancia del aprendizaje significativo, la construcción del conocimiento y la interacción.

La tercera etapa culmina con el Segundo Congreso Universitario (noviembre del 2000), cuando se acuerda que la producción académica debe basarse en la integración y colaboración de tecnologías nuevas y tradicionales, de acuerdo con el alcance, las características y las potencialidades de enseñanza y aprendizaje de cada uno de los diferentes medios de comunicación. A partir de entonces, los materiales didácticos impresos se producen tomando en cuenta que son parte de un conjunto integrado de materiales didácticos y, además, tanto el impreso, como los demás materiales deben basarse en los principios que dictan la misión, visión, proyecto educativo y modelo pedagógico, tales como la interactividad, el aprendizaje independiente, una educación conducente al desarrollo de competencias profesionales, a una formación humanista, reflexiva, crítica, creativa y de compromiso con la sociedad y el medio ambiente.

En síntesis, los profesionales involucrados en la producción de materiales didácticos impresos para la UNED, han ido construyendo pautas para que dichos materiales sean adecuados para el estudio a distancia, favorezcan la formación permanente e involucren a los estudiantes a participar de manera activa en su aprendizaje. 
Giuseppa D `Agostino, Johanna MeZa y Alejandra Cruz

ELEMENTOS Y CARACTERÍSTICAS DEL MATERIAL IMPRESO QUE FAVORECEN LA FORMACIÓN Y EL APRENDIZAJE A DISTANCIA EN LA UNED. SiSTEMATIZACIÓN DE CARACTERÍSTICAS Y RESULTADOS GLOBALES

\section{APORTES REALIZADOS POR FUNCIONARIOS EXPERTOS DE LA UNED DE COSTA RICA SOBRE LAS CARACTERÍSTICAS QUE DEBEN POSEER LOS MATERIALES ESCRITOS Y SISTEMATIZACIÓN Y CONSTRUCCIÓN DE ÁREAS TEMÁTICAS}

Con base en la teoría y en los documentos internos de la UNED sobre la producción de materiales didácticos impresos (resumidos en el punto anterior), se definen tres áreas temáticas o elementos (contenido, didáctica y aspectos técnicográficos) y se confecciona un cuestionario con preguntas abiertas dirigido a un grupo de profesionales que se considera expertos en la temática en cuestión.

A estos profesionales se les solicita que indiquen las características que, en su opinión, aseguran un nivel óptimo de calidad del material escrito para el sistema de estudio a distancia de la UNED, de acuerdo con esas áreas temáticas.

Los profesionales consultados, además de contestar lo que se les solicita, agregan tres áreas más, a saber: discurso expositivo, diseño curricular y estrategias de producción. Estas dos últimas, si bien no son directamente elementos constitutivos del material didáctico impreso, son consideradas por nuestros informantes como factores determinantes de la calidad del material y, por ende, de importancia fundamental en su producción.

La información obtenida se analiza y estructura de manera sistemática por elementos (áreas temáticas), características y atributos, y se elabora un documento enumerativo que constituye un aporte fundamental del proyecto de investigación en su totalidad ${ }^{1}$.

A continuación se exponen las áreas temáticas aludidas, las características de cada área y sus respectivos atributos (subcaracterísticas), así como los factores determinantes de la calidad.

\section{Áreas temáticas, características y atributos del material didáctico escrito \\ Contenido}

Los profesionales que han contestado la encuesta consideran que, en síntesis, un buen material impreso para el autoaprendizaje, en lo referente a contenido, es el que en este aspecto es: actual, riguroso, pluridimensional, crítico, relevante y pertinente con los requerimientos de la disciplina, de la carrera, del aprendizaje independiente $y$ del contexto social. 
Giuseppa D `Agostino, Johanna Meza y Alejandra Cruz

ELEMENTOS Y CARACTERÍSTICAS DEL MATERIAL IMPRESO QUE FAVORECEN LA FORMACIÓN Y EL APRENDIZAJE A DISTANCIA EN LA UNED. SiSTEMATIZACIÓN DE CARACTERÍSTICAS Y RESULTADOS GLOBALES

Respetando las respuestas aportadas, se conforman las siguientes categorías de características y sus respectivos atributos o subcaracterísticas.

- Actualización

o Material actualizado

o Material rico del área de estudio

o Bibliografía actualizada

o Producción con base en temas actuales

o Fuentes actualizadas

o Contextualización del tema hacia la vida nacional y/o latinoamericana

- $\quad$ Rigurosidad

o Solidez de los argumentos

o Inducción hacia el pensamiento claro y riguroso

o Exactitud

o Veracidad

o Ausencia de ambigüedad en el texto

o Ausencia de errores conceptuales

o Nomenclatura y simbología propias del contenido de estudio

o Información correcta, válida y oportuna

- $\quad$ Pluridimensionalidad

o Desarrollo de un enfoque integral de la asignatura

o Aporte de bibliografía básica y complementaria

o Planteamiento de diferentes posiciones, puntos de vista y orientaciones posibles para conocer y aplicar el tema

o Presentación de visiones con carácter interdisciplinario y transdiciplinario

o Favorecimiento de la formación integral del estudiante

o Presentación de distintos enfoques y orientaciones

- Relevancia y criticidad

o Exposición de los aspectos más importantes de la materia en cuestión

o Excelencia académica

o Indicación de la importancia de los contenidos expuestos dentro del campo disciplinario y profesional

o Contenidos potencialmente significativos y estimulantes 
Giuseppa D `Agostino, Johanna MeZa y Alejandra CRuZ

ELEMENTOS Y CARACTERÍSTICAS DEL MATERIAL IMPRESO QUE FAVORECEN LA FORMACIÓN Y EL APRENDIZAJE A DISTANCIA EN LA UNED. SiSTEMATIZACIÓN DE CARACTERÍSTICAS Y RESULTADOS GLOBALES

o Cuestionamientos sobre la realidad social y contextual

o Posiciones críticas distintas

- Pertinencia

o Contenidos y diseño didáctico coherentes con los propósitos de la carrera y del curso

o Contenidos y actividades educativas suficientes y consistentes

o Consistencia entre los distintos elementos del material con los objetivos del curso

o Contenidos enlazados con conocimientos previos de los estudiantes

o Nivel de dificultad correspondiente al nivel de la carrera y a la naturaleza del curso

o Nivel de complejidad adecuado al nivel de los estudiantes

o Equilibrio en la densidad de lo expuesto

o Balance adecuado entre lo propuesto y lo desarrollado

o Densidad, profundidad y complejidad acorde con el nivel y la naturaleza del curso

\section{Discurso expositivo}

Los profesionales que han contestado la encuesta apuntan a la necesidad de que el discurso expositivo de un texto didáctico debe ser de calidad. Para ello evidencian las siguientes características y atributos.

- Claridad

o Contenido claro y agradable

o Texto sencillo y conciso

o Texto con indicaciones precisas

o Mensaje directo "que vaya aI grano"

o Lenguaje apropiado y accesible al estudiante

o Utilización de citas claras

o Lenguaje claro y preciso

o Discurso fluido

- Estilo

o Estilo culto

o Estilo sobrio y académico en la presentación del material impreso

o Buena calidad expositiva que promueva construcción y manipulación de 
Giuseppa D `Agostino, Johanna Meza y Alejandra CRuZ

ELEMENTOS Y CARACTERÍSTICAS DEL MATERIAL IMPRESO QUE FAVORECEN LA FORMACIÓN Y EL APRENDIZAJE A DISTANCIA EN LA UNED. SiSTEMATIZACIÓN DE CARACTERÍSTICAS Y RESULTADOS GLOBALES

conceptos

o Redacción amena

o Lenguaje académico coloquial

o Escrito agradable, interesante y motivador

o Uso de lenguaje apropiado a la especialidad

- Orden y coherencia

o Desarrollo de los contenidos en un orden lógico

o Presentación ordenada y coherente del material

o Coherencia interna en la exposición del contenido

- Redacción y ortografía

o Manejo ortográfico perfecto

o Uso correcto de los diferentes tipos de oraciones que existen

o Buena redacción

o Uso pertinente de los extranjerismos

o Uso correcto del español

o Presentación de traducciones correctas, cuando tal recurso sea del caso

\section{Didáctica}

En lo referente a la parte didáctica, los profesionales encuestados evidencian la importancia de la estructura y organización del texto, del uso de métodos que permitan enseñar y aprender con eficacia, y de la aplicación de recursos y estrategias didácticas que clarifican los contenidos y permiten las interrelaciones con el contexto y la experiencia previa del estudiante.

Además, se esmeran en enfatizar la necesidad de motivar al estudiante, orientarlo en el estudio e involucrarlo activamente en la construcción y aplicación del conocimiento. Para ello, sugieren las siguientes características con sus respectivos atributos.

- Diseño, organización y estructura

o Estructura visual y conceptual apropiadas

o Equilibrio entre la parte comunicativa, didáctica, lingüística y técnicográfica

o Equilibrio entre la cantidad y densidad de lo expuesto

o Diseño didáctico 
Giuseppa D `Agostino, Johanna Meza y Alejandra Cruz

ELEMENTOS Y CARACTERÍSTICAS DEL MATERIAL IMPRESO QUE FAVORECEN LA FORMACIÓN Y EL APRENDIZAJE A DISTANCIA EN LA UNED. SISTEMATIZACIÓN DE CARACTERÍSTICAS Y RESULTADOS GLOBALES

o Reproducción lo más fiel posible de una lección presencial

o Aplicación de los principios pedagógicos del paradigma que adopte la UNED para el desarrollo del proceso educativo

o Utilización de métodos y técnicas apropiadas para la educación de adultos, y para el estudio a distancia

o Consideración de los conocimientos y de las experiencias de los estudiantes

o Organización lógica y secuencial del contenido y de las experiencias educativas

o Enlace entre los temas que se inician y los anteriores

- Enfoque y tratamiento didáctico

o Fomento del autoaprendizaje

- Empleo de una metodología y un diseño didáctico que promuevan, faciliten y orienten el estudio y el aprendizaje independiente y permanente

- Utilización de una metodología acorde con las necesidades y características del estudiante (adulto, trabaja y no asiste a clases)

- Sugerencias sobrela manera en que el estudiantepuededesenvolverse en el estudio del material: guía y aporte de ideas en torno al uso del tiempo, a las estrategias para realizar las tareas y los trabajos investigativos, a las formas de evaluar su aprendizaje, etc.

- Descripción detallada de los procesos que, por su naturaleza, no son fáciles de entender

o Motivación

- Producción de materiales atractivos

- Estimulo de la creatividad

- Texto capaz de mantener la atención e interés

- Creación de ambientes propicios para que el estudiante aprenda

- Estimulo para que el estudiante utilice todos los recursos que se presentan en un material

- Utilización de textos con propuestas formativas ricas en elementos aplicables al contexto y generadoras de aprendizaje permanente

- Evitación de textos densos, aburridos y confusos

o Interactividad

- Elaboración del material desde la perspectiva de un modelo interactivo de comunicación-educación, que propicie una conversación didáctica 
Giuseppa D `Agostino, Johanna Meza y Alejandra Cruz

ELEMENTOS Y CARACTERÍSTICAS DEL MATERIAL IMPRESO QUE FAVORECEN LA FORMACIÓN Y EL APRENDIZAJE A DISTANCIA EN LA UNED. SiSTEMATIZACIÓN DE CARACTERÍSTICAS Y RESULTADOS GLOBALES

- Diálogo constante con el estudiante

- Utilización del enfoque dialéctico

- Uso de propuestas o arreglos instructivos centrados en la actividad del alumno

- Formulación de preguntas retadoras al inicio y durante el desarrollo de todo el texto

o Criticidad

- Presentar contenidos problematizadoresy retadores delainteligencia del estudiante

- Estimular los procesos mentales superiores

- Propiciar el aprendizaje por descubrimiento y la creatividad

- Despertar el interés, la curiosidad, el asombro, la reflexión, la imaginación, la memoria, la solución de problemas y la activación de esquemas mentales

- Motivar al estudiante para replantearse los contenidos

- Permitir la construcción de conocimiento por parte del estudiante

- Estimular al estudiante para la investigación y búsqueda del conocimiento mediante el uso de otras fuentes

- Presentar en el material posibles relaciones con otras disciplinas

o Aplicación y práctica

- Inclusión cuando se amerite, de un diagnóstico inicial para que el estudiante mida cuánto sabe de la materia

- Favorecimiento de la evaluación del aprendizaje

- Uso de evaluaciones formativas y sumativas

- Presentación de ejemplos y analogías

- Inclusión de ejercicios de autoevaluación de buena calidad y cantidad

- Presentación de ejercicios con su respectiva respuesta o criterios para que el estudiante revise y reoriente su aprendizaje

- Inclusión de preguntas de desarrollo y problemas que exijan soluciones complejas, y que permitan avanzar y construir a partir de lo aprendido

- Uso de ejercicios que agilicen la comprensión de los temas y que vayan de menor a mayor nivel de dificultad

- Uso de ejercicios que evalúen el dominio de los contenidos instrumentales del curso, pero sin evaluar aspectos muy puntuales ni promover la repetición de contenidos 
Giuseppa D `Agostino, Johanna MeZa y Alejandra CRuZ

ELEMENTOS Y CARACTERÍSTICAS DEL MATERIAL IMPRESO QUE FAVORECEN LA FORMACIÓN Y EL APRENDIZAJE A DISTANCIA EN LA UNED. SiSTEMATIZACIÓN DE CARACTERÍSTICAS Y RESULTADOS GLOBALES

- Planteamiento de actividades de aprendizaje complementarias, integrativas y de aplicación de los conceptos estudiados

- Presentación de prácticas y trabajos de investigación

- Favorecimiento mediante las actividades complementarias de procesos de inferencia de conceptos y cuestionamientos a partir de la propia realidad del estudiante

- Estimulo, orientación, enriquecimiento y control del aprendizaje del estudiante mediante todos los tipos de evaluación de los aprendizajes que se presenten en el material

- Recursos y apoyos didácticos

o Objetivos

- Objetivos generales y específicos por tema

- Objetivos claros, críticos y creativos

- Temas desarrollados según los objetivos generales y específicos

- Objetivos que no fomenten un aprendizaje memorístico ni fragmentado para no perder de vista la integración de los contenidos

- Aclaraciones acerca de lo que el estudiante es capaz de hacer antes y después de utilizar el material escrito

- Consideración delos objetivos en la autoevaluación, en las actividades y en las experiencias educativas propuestas

o Ayudas visuales

- Usar el índice en los textos

- Incluir un diccionario de palabras clave

- Explicar los términos técnicos o propios de la disciplina tratada, así como utilizar nomenclatura, simbología y expresiones típicas del tema en estudio

- Emplear ilustraciones, gráficos, cuadros y tablas (si esto se requiere) para contribuir con la aclaración del texto

- Utilizar colores y negrita para destacar cosas importantes

- Utilizar varios tipos de letra de acuerdo con las diferentes situaciones

- Usar técnicas que resalten conceptos de interés y básicos para el conocimiento, así como las definiciones o los contenidos más importantes

- Brindar espacio para que los estudiantes hagan anotaciones y síntesis 
Giuseppa D`Agostino, Johanna MeZa y Alejandra CRuZ

ELEMENTOS Y CARACTERÍSTICAS DEL MATERIAL IMPRESO QUE FAVORECEN LA FORMACIÓN Y EL APRENDIZAJE A DISTANCIA EN LA UNED. SiSTEMATIZACIÓN DE CARACTERÍSTICAS Y RESULTADOS GLOBALES

- Bibliografía

o Distinguir bibliografía de referencia de bibliografía de consulta

o Comentar bibliografía básica

o Correlacionar el material con otros textos

o Recomendar lecturas específicas para cada contenido

o Propiciar acceso a diferentes servicios bibliográficos

- $\quad$ Síntesis y resúmenes

o Sintetizar ideas principales

o Incluir síntesis finales

o Elaborar buenos esquemas-resúmenes

o Enseñar al estudiante a elaborar sus propias síntesis

o Prever espacios para anotaciones y síntesis de los estudiantes

\section{Aspectos técnico-gráficos}

En cuanto a los aspectos técnico-gráficos, los profesionales insisten en una "buena edición”, con letra nítida, papel adecuado y con recursos ilustrativos pertinentes y atractivos. Para ello se refieren a las siguientes características y atributos:

- $\quad$ Ilustraciones y esquemas

o Utilizar recursos ilustrativos de acuerdo con el tema: ilustraciones, gráficos, dibujos, cuadros, caricaturas, fotos, mapas, esquemas, resúmenes, recuadros

o Utilizar recursos ilustrativos que sean:

- claros

- con explicaciones

- bien elaborados

- agradables

- de buen tamaño

- amplios y

- de colores

o Uso pertinente de la ilustración visual y gráfica

o Uso proporcional de las ayudas visuales

- $\quad$ Letra

o Tamaño adecuado

o Variedad de tipos de letras para los títulos, subtítulos, definiciones 
Giuseppa D `Agostino, Johanna MeZa y Alejandra CRuZ

ELEMENTOS Y CARACTERÍSTICAS DEL MATERIAL IMPRESO QUE FAVORECEN LA FORMACIÓN Y EL APRENDIZAJE A DISTANCIA EN LA UNED. SiSTEMATIZACIÓN DE CARACTERÍSTICAS Y RESULTADOS GLOBALES

o Variedad de tipos de letra según sea el caso

o Buena tipografía

o Tamaño adecuado del escrito

o $\quad$ Nitidez de la letra

o Tamaño adecuado de páginas

o Suficiente espacio para hacer anotaciones

- Papel

o Utilizar papel liviano, bond y de calidad

o Usar hojas blancas

o Cuidar el brillo de la hoja

o No usar papel periódico

- Calidad de la edición

o Presentación agradable

o Impresión a colores

o Impresión de buena calidad

o Portada agradable

o Texto bien encuadernado

o Tamaño de manuscrito adecuado y manejable, que facilite la manipulación

o Alta calidad de la edición

o Buena presentación

o Adecuado formato

o Buena impresión

o Relación costo-edición

o No uso de textos fotocopiados

o Edición cuya lectura no cause cansancio

o Material no recargado de texto

\section{Factores que inciden en la calidad del material didáctico escrito}

Los profesionales que contestan la encuesta hacen énfasis en la necesidad de un planeamiento curricular que integre y articule los distintos componentes de la oferta educacional de los programas y de los cursos respectivos. También, ponen de manifiesto la conveniencia de un trabajo de equipo ya sea a nivel de planeamiento como de producción de materiales. Sugieren, además, la revisión periódica de los materiales escritos para evitar su caducidad y desactualización, y abogan por la 
Giuseppa D `Agostino, Johanna Meza y Alejandra Cruz

ELEMENTOS Y CARACTERÍSTICAS DEL MATERIAL IMPRESO QUE FAVORECEN LA FORMACIÓN Y EL APRENDIZAJE A DISTANCIA EN LA UNED. SiSTEMATIZACIÓN DE CARACTERÍSTICAS Y RESULTADOS GLOBALES

puesta en práctica de criterios y procedimientos de producción dentro de una línea unitaria. Para ello, se refieren a los siguientes aspectos:

\section{Diseño curricular}

- Planificación

o Planeamiento detallado e integrado

o Planeamiento de carreras y cursos correspondiente a la naturaleza del área disciplinaria y profesional, a la modalidad a distancia y a las características y necesidades de la población destinataria

o Materiales y recursos didácticos que respondan a las necesidades y a los requisitos del perfil ocupacional y profesional de la carrera

o Relación de los contenidos con género, medio ambiente y valores

o Ubicación adecuada de los cursos y sus componentes con respecto al plan de estudios

o Trabajo de equipo

- Integración de los distintos recursos didácticos y conformación de una propuesta de formación unitaria y diversificada

o Interrelación entre el material escrito con los otros medios y mediaciones que hacen parte del curso

o Consideración de varias formas y medios de apoyo en el aprendizaje

o Consideración de los medios electrónicos

o Uso de todos los recursos didácticos que se requieren

\section{Procedimientos de producción}

- Normas

o Contar con la aprobación de la Dirección de Producción de Materiales Didácticos para la publicación de los materiales didácticos

o Eliminar duplicidad de funciones entre la Oficina de Unidades Didácticas y la Editorial

o Integrar los distintos materiales didácticos y ser revisados por especialistas en contenidos

o Diversificar la forma de producir materiales escritos y combinarlos con otros

o Renovar los materiales cada $3-5$ años 
Giuseppa D `Agostino, Johanna MeZa y Alejandra CRuZ

ELEMENTOS Y CARACTERÍSTICAS DEL MATERIAL IMPRESO QUE FAVORECEN LA FORMACIÓN Y EL APRENDIZAJE A DISTANCIA EN LA UNED. SISTEMATIZACIÓN DE CARACTERÍSTICAS Y RESULTADOS GLOBALES

o Definir normas y procedimientos homogéneos a la hora de producir un material

o Usar descripciones curriculares actualizadas y respondientes a las necesidades del aprendizaje independiente, para elaborar los textos y otros materiales de los cursos

o Tener criterios uniformes para el manejo de los contenidos por parte de los productores académicos

o Hacer buena escogencia del autor

- Trabajo interdisciplinario y en equipo

o Trabajo en equipo de profesionales (productores, especialista en contenido y en diseño editorial, encargado de la carrera y de la cátedra, filólogo, autor calificado)

o Intervención de profesionales con conocimientos en el campo de la educación de adultos y en educación a distancia

o Autor apoyado por equipo de expertos en la elaboración de materiales para educación a distancia

En síntesis, los profesionales consultados subrayan que si bien no hay una "receta" para lograr un óptimo material didáctico para el auto-aprendizaje, sí hay rasgos que lo caracterizan como tal. Entre estos, resaltan tanto una visión actualizada, pertinente, rica y de sólidos argumentos del área de estudio, así como una buena presentación física del material y un discurso expositivo de calidad. También, señalan la importancia de motivar para el estudio al aprendiz y de guiar y promover su actividad constructiva, su reflexión, creatividad y autorregulación.

Lo anterior lo condicionan a una óptima labor interdisciplinaria de preparación y diseño curricular tanto de los programas como de los cursos y de las diversas experiencias de aprendizaje que estos requieren para su aplicación en un sistema educativo a distancia.

Abocan por la diversificación en las formas de producir materiales didácticos y, dentro de cada una de estas, por procedimientos de línea unitaria y por el uso de distintos medios y materiales integrados y estructurados para el logro de un fin común. 
Giuseppa D `Agostino, Johanna Meza y Alejandra CRuZ

ELEMENTOS Y CARACTERÍSTICAS DEL MATERIAL IMPRESO QUE FAVORECEN LA FORMACIÓN Y EL APRENDIZAJE A DISTANCIA EN LA UNED. SISTEMATIZACIÓN DE CARACTERÍSTICAS Y RESULTADOS GLOBALES

\section{APLICACIÓN PRÁCTICA DE LA SISTEMATIZACIÓN DE LOS RESULTADOS ANTERIORES: EL CUESTIONARIO A ESTUDIANTES Y SUS RESULTADOS GENERALES}

Partiendo del análisis documental sobre la producción de materiales escritos para la educación a distancia; del análisis de los documentos internos de la UNED, y de la sistematización de las áreas temáticas con sus respectivas características y atributos (efectuada a partir de la información obtenida de los profesionales considerados como expertos en educación a distancia) y de la información aportada por los graduados que se entrevistaron ${ }^{2}$, se infiere y elabora una clasificación de aquellos aspectos de las cuatro áreas temáticas sobre los materiales escritos, de mayor interés para ser valorados con los estudiantes. Esto, a razón de que no todos los elementos y las características enlistadas son aspectos de directa competencia de ellos.

Las cuatro grandes áreas temáticas de interés fueron: CONTENIDO, ENFOQUE METODOLÓGICO, RECURSOS DIDÁCTICOS y RECURSOS GRÁFICOS. Para cada una de ellas se seleccionaron las características con los atributos (subcaracterísticas) que las tipifican, y luego se procedió a construir un promedio de once preguntas cerradas para cada una de las áreas temáticas consideradas en el cuestionario. ${ }^{3}$

Antes de aplicar masivamente el cuestionario a los estudiantes, este fue valorado por los mismos expertos directamente involucrados en la producción de materiales y que fueron parte del conjunto de profesionales entrevistados con antelación; luego se probó tres veces con pequeños grupos de estudiantes. El instrumento se aplicó durante los segundos exámenes ordinarios del primer cuatrimestre del 2002 a una muestra de 1900 estudiantes y los datos se procesaron en SPSS.

A continuación se presentan e interpretan los resultados más destacados desde un punto de vista global, que se desprenden de la totalidad de las respuestas obtenidas en cada una de las características contempladas en las cuatro áreas de los materiales impresos que se plantean en el cuestionario aludido. Por razones metodológicas y de espacio se dejan para la siguiente publicación, el análisis y las conclusiones que se desprenden del cruce entre estos resultados y las variables que tipifican a los estudiantes: escuela, nivel de carrera, edad, centro universitario, sexo, estado civil y condición laboral. 
Giuseppa D `Agostino, Johanna MeZa y Alejandra CRuZ

ELEMENTOS Y CARACTERÍSTICAS DEL MATERIAL IMPRESO QUE FAVORECEN LA FORMACIÓN Y EL APRENDIZAJE A DISTANCIA EN LA UNED. SiSTEMATIZACIÓN DE CARACTERÍSTICAS Y RESULTADOS GLOBALES

\section{Resultados generales sobre el perfil del estudiante}

Antes de ofrecer los principales datos obtenidos de la aplicación del cuestionario, es importante señalar algunos datos relevantes sobre la selección de los estudiantes. $\mathrm{Al}$ respecto, se consideró importante que los estudiantes tuvieran experiencia en el estudio a distancia; por ello se seleccionaron cursos del nivel de bachillerato y se excluyeron los cursos del programa de Estudios Generales, por ser estos, en teoría, estudios iniciales. También, se excluyeron aquellos estudiantes de los talleres de investigación, prácticas, cursos especializados y de posgrado, debido a que muchos de ellos ingresan a las licenciaturas con bachilleratos obtenidos en otras casas de enseñanza y, por lo tanto, sin experiencia acumulada en educación a distancia.

Los datos obtenidos perfilan un estudiante con características similares a las que arrojan los anuarios estadísticos. Las diferencias que se registran son consecuencia de la premeditada selección. Por ejemplo, dado que no se seleccionaron estudiantes de primer ingreso, la muestra presenta a estudiantes con edades promedio mayores que las reflejadas en los datos de los anuarios para la población total. Pero la muestra evidencia una población predominantemente femenina tanto por nivel como por escuela, laboralmente activa y con alguna relación de pareja (casados, separados, unión libre o divorciados). Características, todas ellas, similares a las que denota el patrón del perfil del estudiante unediano delineado en los anuarios estadísticos de la Institución.

Tanto en la muestra como en la población total, Educación es la escuela donde se concentra la mayor población estudiantil, y además, mayoritariamente femenina, con un porcentaje de $85 \%$ en la muestra, muy por encima del promedio general $(70,3 \%$, Anuario Estadístico). Esto es congruente con la situación que ha tenido la UNED desde su nacimiento, pues la mayoría de sus estudiantes se sitúan en Educación y estos, a su vez, son de sexo femenino.

\section{Contenido}

A continuación se presenta el cuadro 1 donde se evidencian los resultados obtenidos por cada uno de los rubros que, sobre contenido, se incluyen en el cuestionario aplicado a los estudiantes para conocer su opinión sobre la importancia que, en orden prioritario ellos le adjudican a esos rubros para su formación académica y profesional. 
Giuseppa D `Agostino, Johanna Meza y Alejandra CRuz

ELEMENTOS Y CARACTERÍSTICAS DEL MATERIAL IMPRESO QUE FAVORECEN LA FORMACIÓN Y EL APRENDIZAJE A DISTANCIA EN LA UNED. SiSTEMATIZACIÓN DE CARACTERÍSTICAS Y RESULTADOS GLOBALES

\section{Cuadro 1}

Características del contenido de las unidades didácticas según importancia para la formación académica y profesional atribuida por los estudiantes encuestados

(Valores relativos)

1. Se relacionan con los propósitos y objetivos de formación de la carrera y del curso.

2. Se relacionan con la realidad nacional, el ámbito laboral y profesional.

3. Promueven la participación del estudiante y lo incentivan a reflexionar, investigar, explorar, aplicar y a tomar posición crítica frente a lo que aprende.

4. Presentan descripciones precisas, concretas y específicas de los conceptos que se aportan.

5. Estimulan y propician, en el estudiante, el proceso de humanización, de formación de valores y de respeto al medio ambiente.

6. Presentan contenidos y bibliografías actualizadas.

7. Plantean y fundamentan los diferentes enfoques y posiciones del área disciplinaria de que se trate.

8. Se relacionan con la experiencia cultural y nivel de conocimiento del estudiante.

9. Privilegian la repetición de definiciones, principios, conceptos e ideas aportadas.

10. Requieren ser complementados por el estudiante mediante el estudio y la consulta de materiales adicionales y la realización de actividades adicionales.

11. Son abundantes y reiterativos sobre los conceptos desarrollados. 
Giuseppa D `Agostino, Johanna MeZa y Alejandra CRuZ

ELEMENTOS Y CARACTERÍSTICAS DEL MATERIAL IMPRESO QUE FAVORECEN LA FORMACIÓN Y EL APRENDIZAJE A DISTANCIA EN LA UNED. SISTEMATIZACIÓN DE CARACTERÍSTICAS Y RESULTADOS GLOBALES

Como se puede ver en el cuadro anterior, las dos principales características sobre el contenido de las unidades didácticas consideradas por los estudiantes de mayor importancia para su formación académica y profesional son, por una parte, (77, 32\%), la concordancia entre los contenidos desarrollados en los materiales y los propósitos de formación de la carrera y del curso y, por otra parte, casi en un mismo nivel de importancia, la preferencia se direcciona $(70,37 \%)$ hacia aquellos contenidos estrechamente relacionados con la realidad nacional y la actividad laboral y profesional. Las dos preferencias encuentran asidero tanto en las predilecciones manifestadas por los graduados que se entrevistaron, como en las preocupaciones de los profesionales de la UNED que se encuestaron y en los lineamientos de la institución. También, son muy coherentes, en particular la segunda, con el interés general del estudiante adulto de vincular lo que aprende con su rol social y con su realidad y actividad laboral y profesional.

Desde aquí es interesante resaltar esta exigencia de los estudiantes de contar con experiencias educativas en las cuales la formación en competencias directamente ligadas con las prácticas profesionales y laborales tenga un peso mayor. El estudiante, como colectivo, percibe un amplio vacío en este sentido, dentro de las ofertas académicas de la institución. He aquí un reto para las entidades relacionadas con el diseño, la elaboración, la implementación y la evaluación de las experiencias académicas, pues en estas labores dichas entidades, además del conocimiento conceptual, deberán considerar, de manera más consciente, el conocimiento y las prácticas procedimentales y valorativas.

Los dos siguientes aspectos del contenido con una importancia considerable, aunque no tan alta como los dos anteriormente señalados, se refieren a los "contenidos que involucran e incentivan al estudiante a reflexionar, investigar, explorar, aplicar y a tomar posiciones críticas frente a lo que aprende" (59,89\%) y "Contenidos con descripciones precisas, concretas y específicas de los conceptos que se aportan" seleccionada por el 56,89\% de los estudiantes encuestados. He aquí, una posición clara del estudiante con respecto a su interés en participar activamente en su propia experiencia de aprendizaje.

Así mismo, el estudiante (50,58\%) estima valiosos los contenidos que incentivan el proceso de humanización, formación de valores y respeto al medio ambiente. Este es también un aspecto de interés, no solo para considerarse en el nivel de los materiales didácticos sino de ser tomado en cuenta para el diseño y el planeamiento curricular de la oferta académica de la Institución. He aquí un desafío para la UNED: integrar estos ejes transversales en sus propuestas curriculares y sobre todo determinar y 
Giuseppa D `Agostino, Johanna Meza y Alejandra Cruz

ELEMENTOS Y CARACTERÍSTICAS DEL MATERIAL IMPRESO QUE FAVORECEN LA FORMACIÓN Y EL APRENDIZAJE A DISTANCIA EN LA UNED. SISTEMATIZACIÓN DE CARACTERÍSTICAS Y RESULTADOS GLOBALES

utilizar aquellos recursos, intervenciones y estrategias didácticas interdisciplinarias que son más aptas para su implementación en la práctica educativa contextual de la Universidad.

Se observa además, que el estudiante, al igual que los graduados y los profesionales de la UNED, le confiere un buen grado de importancia $(45,80 \%)$ a la actualidad y vigencia de los contenidos y de la bibliografía de los materiales.

El aspecto que los estudiantes privilegian menos como elemento importante para su formación es la presencia de contenidos abundantes y reiterativos (el 82\% rechaza este rubro).

Coinciden aquí las opiniones de graduados y estudiantes que prefieren materiales didácticos con contenidos que no divaguen y vayan al "grano" del asunto y a la sustancia de la propuesta educativa. Se evidencia también un alto rechazo (75\%) hacia aquellos materiales cuyos contenidos privilegian aprendizajes de repetición.

El 69,58\% de los estudiantes no manifiesta preocupación por los contenidos que se relacionen con su experiencia cultural y su nivel de conocimiento. Esto, a nuestro aviso, es una respuesta engañosa pues si cotejamos esta contestación con la registrada en el rubro de "Contenidos relacionados con el contexto, nacional y laboral" percibimos que el estudiante, al contestar positivamente este último $(73,37 \%)$ se abstuvo, tal vez, de seleccionar la primera (solo podía marcar 5 características). Es posible que haya considerado que un contenido estrechamente vinculado con la realidad nacional también se vincula con su propia experiencia cultural. Confirman esta suposición la escogencia que los estudiantes $(57,35 \%)$ hacen del segundo rubro del enfoque metodológico (ver cuadro 2), relativo a la importancia de iniciar el proceso instructivo a partir de los conocimientos que el estudiante maneje.

Los resultados indican también que el 66\% de los estudiantes privilegian poco la pluridimensionalidad del contenido que, es un punto preferencial en la propuesta de nuestros profesionales encuestados. En general percibimos que el estudiante no considera óptimo para él y su situación de adulto el uso de materiales extensos y amplios.

Asimismo, llama la atención el resultado registrado con respecto a aquellos materiales cuyos contenidos requieren ser complementados mediante el estudio y la consulta de materiales adicionales y la realización de actividades extra. Este resultado puede inducir a pensar que el estudiante no desea involucrarse activamente en su 
Giuseppa D `Agostino, Johanna MeZa y Alejandra Cruz

ELEMENTOS Y CARACTERÍSTICAS DEL MATERIAL IMPRESO QUE FAVORECEN LA FORMACIÓN Y EL APRENDIZAJE A DISTANCIA

EN LA UNED. SiSTEMATIZACIÓN DE CARACTERÍSTICAS Y RESULTADOS GLOBALES

aprendizaje; sin embargo, consideramos que esta conclusión sería, en parte, equívoca. Ello porque el $60 \%$ de esos mismos estudiantes privilegian, para su formación, los materiales cuyos contenidos los involucran a trabajar de manera activa, es decir, reflexionando, investigando y explorando. Es factible, que los que escogieron este último rubro, no incluyeron en la lista de su preferencia el anterior. Además, considerando también los aportes y las preferencias de los graduados, percibimos en esta respuesta relativa a las actividades extra y a la consulta de materiales adicionales, el deseo del estudiante (es un adulto, trabaja, tiene otras ocupaciones y tiempo limitado) de ser involucrado de una manera activa dentro del conjunto de experiencias y actividades de aprendizaje contempladas en los materiales que constituyen el acervo de los cursos, pero no de ser involucrado en actividades y tareas que exceden dichos materiales y le requieren tiempo y dedicación extra.

En torno a esta posición estudiantil hay que buscar respuestas. Abrir espacios de reflexión e investigación sobre los factores que la determinan y la influencia que ha tenido, en el estudiante, la metodología de trabajo de la UNED; surgen al respecto algunas interrogantes: ¿Cuán largo ha llegado la dependencia del estudiante respecto del "paquete del curso"?; ¿cuánto han influido, en sus modos y formas de aprender, las prácticas de enseñanza y de evaluación que la UNED efectúa?; ¿cuán integradas están esas actividades y tareas adicionales con la propuesta pedagógica de cada curso en que se plantean?; ¿qué orientaciones y apoyos se le dan al estudiante, para poder realizar las actividades y tareas exigidas?; ¿qué tan autónomo y capacitado para el "aprender a aprender" logra ser el estudiante de la UNED?

\section{Enfoque Metodológico}

A continuación, se aporta el cuadro 2, donde se pueden apreciar las preferencias de los estudiantes encuestados acerca de las características metodológicas que ellos consideran de mayor relevancia para su aprendizaje independiente.

\section{Cuadro 2}

Aspectos del enfoque metodológico de las unidades didácticas según la utilidad para aprender, atribuida por los estudiantes encuestados (valores relativos) 
Giuseppa D `Agostino, Johanna Meza y Alejandra CRuZ

ELEMENTOS Y CARACTERÍSTICAS DEL MATERIAL IMPRESO QUE FAVORECEN LA FORMACIÓN Y EL APRENDIZAJE A DISTANCIA EN LA UNED. SISTEMATIZACIÓN DE CARACTERÍSTICAS Y RESULTADOS GLOBALES

\section{ENFOQUE METODOLÓGICO}

Total

1. Formulación de preguntas y ejercicios que inviten a reflexionar a lo largo del desarrollo del tema.

2. Repaso de los conocimientos previos.

3. Orientación sobre la forma de estudiar los distintos temas.

4. Criterios e indicadores para que el estudiante autoevalúe sus respuestas a los ejercicios planteados.

5. Estímulo para que el estudiante reformule y aporte ideas, conceptos y principios.

6. Orientaciones para que el estudiante elabore sus propios resúmenes.

7. Propuesta de actividades que permitan integrar y aplicar los contenidos.

8. Uso de anécdotas y analogías

9. Presencia de problemas retadores que permitan ir más allá de lo aprendido con el texto.

10. Lenguaje coloquial.

11. Predominio del aprendizaje reproductivo y memorístico.

Al observar el cuadro aquí presentado sorprende de inmediato que los estudiantes privilegian como el principal aspecto metodológico de mayor utilidad para aprender, la formulación de preguntas y ejercicios que le faciliten la reflexión a lo largo del desarrollo del tema $(66,49 \%)$.

Otros aspectos metodológicos que ellos consideran deayuday apoyo para aprender son, en orden de prioridad: "el repaso de los conocimientos previos" (57,36\%), "una orientación sobre la forma de estudiar los temas" (56,31\%), la "presencia de criterios e indicadores para que el estudiante autoevalúe sus respuestas a los ejercicios que se le proponen en el material didáctico" (54,30\%). También, le dan importancia $(52,72 \%)$ a las oportunidades de que disponen para reformular y aportar conceptos e ideas y para elaborar sus propios resúmenes $(51,72 \%)$.

La preferencia hacia las anteriores características metodológicas coincide con lo manifestado por los graduados que, en términos generales, aprecian las orientaciones 
Giuseppa D `Agostino, Johanna MeZa y Alejandra Cruz

ELEMENTOS Y CARACTERÍSTICAS DEL MATERIAL IMPRESO QUE FAVORECEN LA FORMACIÓN Y EL APRENDIZAJE A DISTANCIA EN LA UNED. SISTEMATIZACIÓN DE CARACTERÍSTICAS Y RESULTADOS GLOBALES

para el estudio, así como las oportunidades y propuestas para un aprendizaje activo con posibilidades de prácticas y ejercicios. Sobre esta misma línea, los aportes teóricos y los de los expertos consultados, apuntan hacia una mediación pedagógica que propicie la construcción del conocimiento y un aprendizaje más significativo.

Entre los aspectos que consideran menos útiles para aprender, los estudiantes sitúan el uso de anécdotas y analogías (35,73\%) y el uso de problemas retadores que exijan ir más allá de lo planteado en los materiales de estudio (29.82\%). Con ello se observa, de nuevo (ver rubro anterior), una resistencia por parte de la mayoría de los estudiantes (más del 70\%) a participar en actividades fuera de las que se plantean en el conjunto de materiales de la propuesta pedagógica en la que están involucrados. Pero, esto no significa, tal como lo vimos con anterioridad, que el estudiante rechace un aprendizaje constructivo y un papel dinámico y productivo dentro de ese proceso, sino que se rehúsa, a nuestro parecer, por limitaciones de tiempo y por su situación propia de estudiante adulto, y tal vez, por costumbre y dependencia excesiva del medio maestro, a efectuar tareas que le requieren tiempo y dedicación adicionales a las directamente integradas en la experiencia formativa que se desarrolla y presenta en los materiales didácticos de los cursos.

\section{Recursos didácticos y gráficos}

Los cuadros 3 y 4 dan razón de los resultados generales obtenidos con respecto a los recursos gráficos y didácticos preferidos por los estudiantes encuestados.

\section{Cuadro 3}

Recursos didácticos de las unidades didácticas según utilidad para estudiar atribuida por los estudiantes encuestados

(valores relativos)

\begin{tabular}{llr}
\cline { 2 - 2 } RECURSOS DIDÁCTICOS & Total \\
\hline 1. & Síntesis de las ideas principales. & 73,14 \\
2. & Presencia de conclusiones por tema. & 54,14 \\
3. & Objetivos específicos y detallados. & 49,92 \\
4. & Respuestas completas a los ejercicios & 49,13
\end{tabular}


5. Ejercicios al final del tema.

47,92

6. Ejemplos detallados.

7. Ejercicios que evalúen aspectos puntuales del texto.

8. Objetivos generales.

9. Ejercicios para que el estudiante resuelva dentro del tema.

10. Ejercicios y actividades de aplicación.

11. Tabla de contenidos detalladas.

12. Glosario (definición de términos técnicos).

13. Lecturas complementarias para ampliar temas.

12,30

\section{Cuadro 4}

Recursos gráficos de las unidades didácticas según utilidad para estudiar atribuida por los estudiantes encuestados

(valores relativos)

\section{RECURSOS GRÁFICOS}
1. Esquemas.
86,95
2. Recursos ilustrativos para destacar las ideas principales del texto.
68,39
3. Uso del color en el texto.
63,69
4. Espacios dentro de la unidad para
55,76 hacer anotaciones.
5. Fotografías e ilustraciones.
6. Varios tipos de letras.
40,91
7. Texto con páginas de tamaño mediano.
33,40
8. Uso del color en las figuras.
9. Texto con páginas de tamaño grande.
30,02
10. Texto con páginas de tamaño pequeño.
14,96 
Giuseppa D `Agostino, Johanna Meza y Alejandra CruZ

ELEMENTOS Y CARACTERÍSTICAS DEL MATERIAL IMPRESO QUE FAVORECEN LA FORMACIÓN Y EL APRENDIZAJE A DISTANCIA EN LA UNED. SiSTEMATIZACIÓN DE CARACTERÍSTICAS Y RESULTADOS GLOBALES

En este contexto, por afinidad y reciprocidad corroborativa, los resultados de las áreas de recursos didácticos y gráficos se presentan en conjunto.

El recurso didáctico que los alumnos consideran más importante y útil para estudiar es la presencia de una "síntesis de las ideas principales", con un porcentaje mucho más elevado en comparación con los demás aspectos, 73,14\%. Esta información se ve reforzada con el 86,95\% que señala los "esquemas" como el recurso gráfico más importante.

También, opinan que para ellos son importantes los dos recursos didácticos relacionados con la "presencia de conclusiones por tema" (54,14\%), y los "objetivos específicos y detallados" (49,92\%) los cuales, al igual que con los aspectos anteriores, se complementan con rubros del área gráfica tales como "recursos ilustrativos para destacar las ideas principales del texto" (68,39\%), "uso del color" (63,26\%) y "espacios dentro de la unidad para hacer anotaciones" (55,23\%).

Por otra parte, los estudiantes brindan poca importancia a la presencia de tablas de contenido detallados (21\%), a la presencia de un "glosario" (17.47\%), a las "lecturas complementarias para ampliar temas" (12.30\%), y a los "textos con páginas de tamaño pequeño" (14.96\%). Todos estos componentes resultan insignificantes al comparar sus resultados con los del resto de los rubros incluidos.

Se observa una preocupación central del estudiante por aquellos recurso gráficos y didácticos que le permiten identificar medulares de la materia objeto de estudio y que le faciliten la obtención de una visión global y de conjunto de la temática estudiada. También, sobresale su exigencia de indicaciones claras y detalladas sobre los aprendizajes que se esperan de él. Se debe tener presente que todo el proceso formativo y las evaluaciones de los aprendizajes que se efectúan en la UNED, giran alrededor de los objetivos, que constituyen el faro orientador por excelencia. Por ende, es consecuente con esto que el estudiante requiera, para dirigir su estudio y práctica educacional, una especificación clara y concreta de los aprendizajes que se esperan de él. En este sentido, es recomendable, en los materiales didácticos, plantear objetivos de aprendizaje claros y precisos y, por otra, introducir, a lo largo del desarrollo de la temática, referencias y actividades de aprendizaje que permitan al estudiante apropiarse de estos objetivos y profundizar la comprensión de sus distintos niveles de exigencia.

A manera conclusiva, los datos antes expuestos brindan información a partir de la cual se pueden efectuar diversas inferencias. Aquí, se destacan las más evidentes y de 
Giuseppa D `Agostino, Johanna Meza y Alejandra Cruz

ELEMENTOS Y CARACTERÍSTICAS DEL MATERIAL IMPRESO QUE FAVORECEN LA FORMACIÓN Y EL APRENDIZAJE A DISTANCIA EN LA UNED. SiSTEMATIZACIÓN DE CARACTERÍSTICAS Y RESULTADOS GLOBALES

mayor significancia para el aprendiz, a saber: en cuanto a contenidos, sobresalen la correspondencia entre estos y los propósitos de formación de la carrera o programa, y su pertinencia contextual y laboral. Ambos aspectos son siempre prioritarios, independientemente de las variables del estudiante (carrera, nivel, sexo, edad, centro universitario, condición laboral y estado civil) con que se crucen. Relevante, también, el lugar preferencial que el estudiante, en general le asigna a una propuesta de formación dirigida hacia el meollo del asunto educacional objeto de aprendizaje y hacia una educación humanista y de valores.

En cuanto al aspecto didáctico es significativa la predilección del estudiante por las oportunidades de orientación pedagógica, por las propuestas de un estudio activo y reflexivo y la incorporación en el material de preguntas, ejercicios y criterios que le permitan afianzar y monitorear su aprendizaje. Además, tiene preferencia por una clara visión de las intenciones educativas (objetivos) y por aquellos recursos didácticos (síntesis, conclusiones y gráficos) que le permitan conformarse una idea de conjunto de la temática objeto de estudio. Con las reservas y las especificidades propias de cada caso, y los matices que emergen desde otras inferencias y puntos de vista, sobre estos datos (ver informe final), en términos amplios, es de nuestra opinión que estas preferencias de los estudiantes son medulares para las consideraciones que a lo interno de la Institución se efectúan para el diseño, la presentación y la mejora de las propuestas educacionales y de los materiales didácticos sobre los cuales estas se apoyan.

\section{REFLEXIÓN FINAL}

Laidentificación delas áreas temáticas delos materiales impresos yla construcción de las características y de los atributos relativos a cada área, permiten contar con una amplia lista afinada y validada de aspectos de interés para la discusión académica y la producción y evaluación de los materiales impresos de la UNED, así como punto referencial en el trabajo con otros tipos de materiales didácticos.

Este documento enumerativo de aspectos de interés, es uno de los aportes medulares del estudio de investigación aquí efectuado. Es a partir de este documento y de la corroboración y afinamiento de sus rubros, mediante entrevistas con graduados de las distintas Escuelas, que se construye y valida, con diferentes grupos de profesionales y alumnos, el cuestionario aplicado a la muestra de 1900 estudiantes. 
Giuseppa D `Agostino, Johanna MeZa y Alejandra CRuZ

ELEMENTOS Y CARACTERÍSTICAS DEL MATERIAL IMPRESO QUE FAVORECEN LA FORMACIÓN Y EL APRENDIZAJE A DISTANCIA EN LA UNED. SiSTEMATIZACIÓN DE CARACTERÍSTICAS Y RESULTADOS GLOBALES

Se insiste aquí en evidenciar que, lo más valioso del proyecto presentado, es el amplio proceso de construcción y reflexión exigido para su puesta en práctica. Se parte desde la revisión teórica y de la experiencia institucional, se sigue con la recopilación y sistematización de los aportes de profesionales con trayectoria en la educación a distancia y se continúa hacia los aportes de opinión de graduados. Todo esto se enlaza a manera de soporte de las estrategias de investigación; las cuales conducen a la obtención de evidencias significativas acerca de las opiniones e ideas que el estudiante de la UNED tiene con respecto a los materiales didácticos que usa a diario para su aprendizaje y formación. Es este proceso sistemático y constructivo, el elemento medular para el éxito de la práctica pedagógica y, es nuestro deseo, que el lector así lo perciba y que la experiencia aquí presentada sea útil para continuar en ese camino.

\section{NOTAS}

$1 \quad$ Una primera versión de este trabajo se entregó a los profesionales consultados y a las instancias directamente involucradas con el quehacer de producción de materiales en el 2001 (CIAC.083-01, 11 de junio, 2001).

2 Los análisis teóricos, de los documentos y de los expertos consultados fueron también la base para planificar una estrategia de recolección de información sobre el tema a graduados unedianos (graduados bachilleres con todos sus estudios universitarios hechos en la UNED). Por motivos de espacio no se entregan en este artículo los aportes de este grupo de informantes.

3 Sobre este particular, la experiencia previa indica que un cuestionario con preguntas cerradas con un máximo de una hoja tamaño carta por ambos lados, permite un buen porcentaje de respuesta, lo cual fue confirmado en el presente estudio.

\section{REFERENCIAS BIBLIOGRÁFICAS}

Barrantes, R. 2000. Educación a distancia: fundamentos. En: Memoria Segundo Congreso universitario UNED. San José, Costa Rica: EUNED, mayo.

Casas Armengol, M. y Stojanovic L. 1999. De Vancouver 1982 a Viena 1999: La educación a distancia toma nuevos rumbos. En: Iberoamericana de Educación a Distancia. Madrid: Vol. 2, $\mathrm{N}^{0}$ 2, pp. 93-112, diciembre.

D’Agostino. G. 200o. Teorías de la Educación a Distancia y sus implicaciones en la producción de materiales didácticos de la UNED. En: Memoria Segundo Congreso universitario UNED. San José, Costa Rica: EUNED, mayo, 210-222.

García Aretio. 2001. La Educación a Distancia: De la teoría a la práctica. Barcelona: Editorial Ariel.

Garrison D. R. 1995. Constructivism and the role of selfinstruccional course material: A Reply. Traducido y publicado en español por Helena 
Giuseppa D `Agostino, Johanna Meza y Alejandra Cruz

ELEMENTOS Y CARACTERÍSTICAS DEL MATERIAL IMPRESO QUE FAVORECEN LA FORMACIÓN Y EL APRENDIZAJE A DISTANCIA EN LA UNED. SiSTEMATIZACIÓN DE CARACTERÍSTICAS Y RESULTADOS GLOBALES

Ramírez. En: Boletín de Resúmenes de publicaciones sobre Educación a Distancia. San José: CIDI, UNED, No 3, 1995 .

Hidalgo, G., Avarado J., D’Agostino, G. y Segura, M. 1998. Indicadores para la puesta en práctica de procesos de autoevaluación en la UNED. San José: UNED, Material impreso, serie $n^{\circ} .2$.

Holmberg, Borge. 1985. Educación a Distancia: Situación y Perspectivas. Buenos Aires: Kapelusz.

1995. The Evaluation of the Character and Practice of Distance Education. Traducido y resumido en español por Helena Ramírez. En: Boletín de Resúmenes de publicaciones sobre Educación a Distancia. San José: CIDI UNED, Nº 3, 1995.

Macdonald, R. M. 1995. The development of printed materials: A view of print production for distance learning in the light of recent developments. En: La Educación a Distancia en Tiempos de Cambios: Nuevas Generaciones, Viejos Conflictos. Proyecto Didáctico Quizón No 123 (Coordinadores: Eustaquio Martín Rodríguez y Manuel Ahijado Quintillán). Madrid: Ediciones de la Torre, 1999.

Meza, J., D’Agostino, G. y Cruz, A. 2003. Elementosy característicasdelmaterial impreso que favorecen la formación y el aprendizaje a distancia en la UNED. San José, Costa Rica: Informe de investigación, Centro de Mejoramiento de los Procesos Académicos (CEMPA) y Programa de Materiales Impresos. Vicerrectoría Académica, UNED. Costa Rica.

Misanchuck, E. R. 1992. Preparing instructional text: Document design using desktop publishing. Englewood Cliffs, N J: Educational Technology Publications.

Rowntree, Derek. 1990 (revised edition). Writing a Self-Instructional Lesson. Teaching Through Self-Instruction. How to Develop Open Learning Materials. London: Kogan Page, 81118.

1998. Assessing the Quality of MaterialsBased Teaching and Learning. Open Learning, June, 12-22.

Universidad Estatal a Distancia (UNED). 1988a. Memoria Primer Congreso Universitario. San José: EUNED, 1 al 5 de febrero. 1988b. Primer Congreso Universitario. Informe de resultado de los talleres. San José: EUNED, junio.

2000. Mociones aprobadas por el Segundo Congreso Universitario. San José: EUNED, febrero.

\section{PALABRAS CLAVE}

Contenidos educativos digitales, Objetos de aprendizaje, Educación a distancia.

\section{KEYWORDS}

Digital Educational Contents, Learning Objetcs, Distance education. 
Giuseppa D `Agostino, Johanna Meza y Alejandra CruZ

ELEMENTOS Y CARACTERÍSTICAS DEL MATERIAL IMPRESO QUE FAVORECEN LA FORMACIÓN Y EL APRENDIZAJE A DISTANCIA EN LA UNED. SiSTEMATIZACIÓN DE CARACTERÍSTICAS Y RESULTADOS GLOBALES

\section{PERFIL ACADÉMICO DEL AUTOR}

Johanna Meza Vargas: Master en Tecnología Educativa. Funcionaria dela Universidad Estatal a Distancia, Costa Rica.

Dirección postal:

Johanna Meza Vargas

Universidad Estatal a Distancia (Costa Rica)

San José, Costa Rica

Teléfono: 2833528

Correo electrónico: jmeza@uned.ac.cr

Fecha de entrega: 14.09.06

Fecha de aceptación: 20.11.06 